\title{
Preparation of Highly Visible-Light Photocatalytic Active N-Doped $\mathrm{TiO}_{2}$ Microcuboids
}

\author{
Kang Zhao*, Zhiming Wu, Rong Tang, and Yadong Jiang \\ State Key Laboratory of Electronic Thin Films and Integrated Devices, School of Optoelectronic Information, \\ University of Electronic Science and Technology of China (UESTC), Chengdu 610054, P.R. China \\ *E-mail: 30300698@qq.com
}

(Received April 3, 2013; Accepted June 26, 2013)

\begin{abstract}
N-doped $\mathrm{TiO}_{2}$ microcuboids were successfully prepared by a simple one-pot hydrothermal method. The samples were characterized by X-ray diffraction, scanning electron microscopy, diffuse reflectance spectroscopy, and $\mathrm{X}$-ray photoelectron spectroscopy. It was found that the $\mathrm{N}$-doped $\mathrm{TiO}_{2}$ microcuboids enhanced absorption in the visible light region, and exhibited higher activity for photocatalytic degradation of model dyes. Based on the experimental results, a visible light induced photocatalytic mechanism was proposed for $\mathrm{N}$-doped anatase $\mathrm{TiO}_{2}$ microcuboids.
\end{abstract}

Key words: Nitrogen-doping, $\mathrm{TiO}_{2}$ microcuboid, Visible photocatalyst, Mechanism

\section{INTRODUCTION}

Titanium dioxide $\left(\mathrm{TiO}_{2}\right)$ is the most widely used photocatalytic material since it exhibits non-toxic nature, high efficiency, chemical inertness, relatively low cost, and photostability. ${ }^{1-4}$ However, the main drawback is that $\mathrm{TiO}_{2}$ photocatalysts can only be excited by ultraviolet (UV) light due to their wide band gap. Therefore, many studies have been devoted to the efficiency improvement of $\mathrm{TiO}_{2}$ under visible light illumination. ${ }^{5-7}$

Over the past twenty years, there has been extensive research into extending the absorption profile of $\mathrm{TiO}_{2}$ into the visible region by doping with main group elements, in particular with nitrogen. Nitrogen doping is one of the simplest synthetic methods to engineer a visible photocatalyst, and it can narrow the band gap of $\mathrm{TiO}_{2}$ to $2.6 \mathrm{eV}$, which consequently gives rise to visible light-driven photocatalytic activity. ${ }^{8}$ Since then, $\mathrm{N}$-doped $\mathrm{TiO}_{2}$ have been prepared by various methods, including sol-gel method, ${ }^{9}$ chemical vapor deposition method, ${ }^{10}$ hydrothermal method ${ }^{11}$ and calcination in ammonia gas. ${ }^{12}$ Among them, the hydrothermal route is an ideal technique for synthesizing $\mathrm{N}$-doped $\mathrm{TiO}_{2}$ nanomaterials with high purity, narrow particle size distribution, controlled morphology and high crystallinity. ${ }^{13}$ For example, Zhao ${ }^{14}$ and his coworkers prepared $\mathrm{N}$-doped $\mathrm{TiO}_{2}$ hollow microspheres by a onestep hydrothermal method, and the prepared $\mathrm{TiO}_{2}$ microspheres showed excellent photocatalytic activities in degrading dyes under visible light irradiation. Liu et al. ${ }^{15}$ prepared N-doped $\mathrm{TiO}_{2}$ nanorods by a simple one-pot sol- vothermal method using hydrazine hydrate and $\mathrm{TiO}_{2}$ colloids as the starting materials, and the sample showed high catalytic activity of decomposing methyl orange and 4chlorophenol under UV and visible light illumination. Despite that several studies have succeeded in the preparation of $\mathrm{N}$-doped $\mathrm{TiO}_{2}$ nanoparticles with various morphologies, no report, so far, synthesized the $\mathrm{N}$-doped $\mathrm{TiO}_{2}$ microcuboids with high visible light photocatalytic activity.

In this article, we report a facile way to obtain $\mathrm{N}$-doped $\mathrm{TiO}_{2}$ microcuboids with high photocatalytic property under $\mathrm{UV}$ and visible light irradiation. It was proven that the prepared $\mathrm{N}$-doped $\mathrm{TiO}_{2}$ microcuboids significantly enhanced the photo absorption abilities and photocatalytic activities as compared to those of $\mathrm{TiO}_{2}$ samples under the same conditions.

\section{EXPERIMENTAL DETAILS}

All reagents were analytical grade and used without further purification. In a typical procedure, $2.37 \mathrm{~g}$ titanium n-butoxide was dissolved into $15 \mathrm{~mL}$ of ethanol. $0.05 \mathrm{~g}$ sodium dodecyl sulfate (SDS) and $2 \mathrm{~mL}$ of ammonia were added into $5 \mathrm{ml}$ of deionized water and stirring continued for $30 \mathrm{~min}$. After sufficient mixing, the mixed solution was added drop wise into the titanium n-butoxide solution with constant stirring. Next, the autoclave was heated at $160{ }^{\circ} \mathrm{C}$ for $12 \mathrm{~h}$ and allowed to cool down naturally. The product was centrifuged, washed several times with distilled water and ethanol, and dried at $60^{\circ} \mathrm{C}$ for $6 \mathrm{~h}$. At last, the sample was then annealed at $450{ }^{\circ} \mathrm{C}$ for $3 \mathrm{~h}$ in air to 
prepare the final N-doped $\mathrm{TiO}_{2}$ microcuboids. For comparison, the $\mathrm{TiO}_{2}$ samples were prepared using the same method.

The phase of the as-prepared products was determined by a Rigaku D/Max $2400 \mathrm{X}$-ray diffractometer (XRD) equipped with graphite monochromatized $\mathrm{CuK} \alpha$ radiation. The morphology of the as-synthesized products was observed directly by a JEOL JSM-5600LV scanning electron microscope (SEM) equipped with energy-dispersive X-ray spectroscopy (EDS). The UV-vis diffuse reflectance spectra (DRS) of the samples over a range of 200-800 nm were collected on a UV-2450 instrument.

The evaluation of photocatalytic activity of the prepared samples for the photocatalytic decomposition of $\mathrm{RhB}$ aqueous solution under $300 \mathrm{~W}$ mercury lamp as a UV source or $300 \mathrm{~W}$ Xe lamp served as a visible-light source with an ultraviolet cutoff filter, which allowed the transmission of visible-light with a wavelength longer than 420 $\mathrm{nm}(\lambda>420 \mathrm{~nm}) .50 \mathrm{mg}$ photocatalyst was dispersed in a $50 \mathrm{~mL}$ aqueous solution of $1.0 \times 10^{-5} \mathrm{M} \mathrm{RhB}$. Before photodegradation, adsorption equilibrium of the dye on catalyst surface was established by mechanical stirring for $30 \mathrm{~min}$. After solar irradiation for some time (30 min), the reaction solution was filtrated to remove the suspended particulates. Then, the concentration of $\mathrm{RhB}$ in solution was analyzed with an UV-visible spectrophotometer (UV-2550, Shimadzu, Japan).

\section{RESULTS AND DISCUSSION}

The typical powders XRD patterns of $\mathrm{TiO}_{2}$ sample and $\mathrm{N}$-doped $\mathrm{TiO}_{2}$ microcuboids are shown in Fig. 1. The crystal structures of the $\mathrm{N}$-doped $\mathrm{TiO}_{2}$ powders represent the anatase phases (JCPDS, card no: 21-1272), which are similar to pure $\mathrm{TiO}_{2}$ sample.

Fig. 2 shows typical SEM images of the $\mathrm{N}$-doped $\mathrm{TiO}_{2}$ microcuboids fabricated by a one-pot hydrothermal method. As shown in Fig. 2a, the overall morphology of the sam-

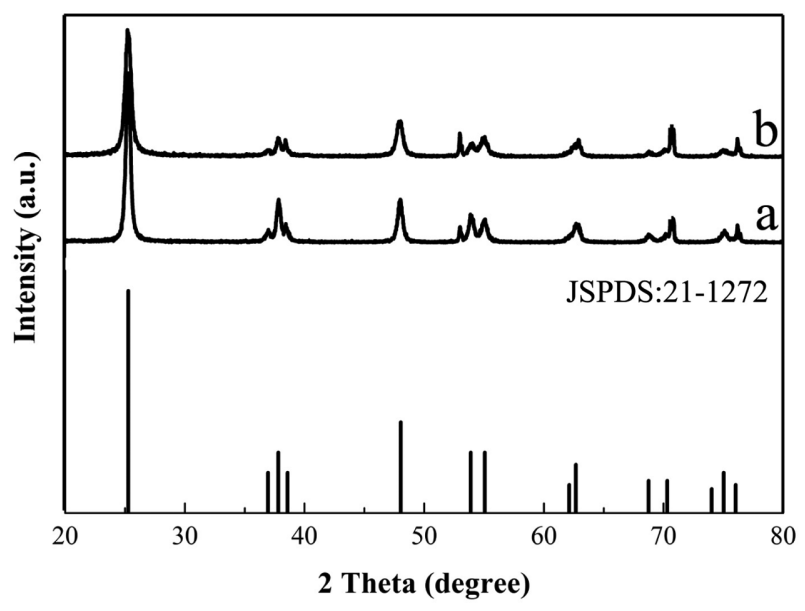

Figure 1. XRD patterns of $\mathrm{TiO}_{2}$ samples (a) and $\mathrm{N}$-doped $\mathrm{TiO}_{2}$ microcuboids (b).

ple indicates that there exists a great deal of uniform cuboidlike microstructures in high yield. The microcuboids are in the length of about $400 \mathrm{~nm}$, in the width of about 300 $\mathrm{nm}$ and in the height of about $250 \mathrm{~nm}$. The corresponding EDS of N-doped $\mathrm{TiO}_{2}$ microcuboids also demonstrates the presence of Ti, $\mathrm{O}$ and $\mathrm{N}$, and the atomic ratio of the $\mathrm{N}$ is about $16 \%$.

Fig. 3 shows the corresponding UV-vis diffuse reflectance spectra of pure $\mathrm{TiO}_{2}$ samples and $\mathrm{N}$-doped $\mathrm{TiO}_{2}$ microcuboids. The absorption edge of pure $\mathrm{TiO}_{2}$ samples is observed at about $380 \mathrm{~nm}$, corresponding to the bandgap energy of $3.2 \mathrm{eV}$. After the $\mathrm{N}$ doping in the $\mathrm{TiO}_{2}$ samples, the absorption spectrum shows that the absorption edge is shifted significantly toward the visible region with a band edge of $535 \mathrm{~nm}(\mathrm{Eg}=2.3 \mathrm{eV})$ (Fig. 3b). The remarkable absorbance for visible light is attributed to the formation of the $\mathrm{O}-\mathrm{Ti}-\mathrm{N}$ linkage, leading to the narrowing of the energy band gap. This phenomenon can also be explained in terms of the formation of an intra-band gap located above the valence band, due to substitution of oxide centers by nitride centers and/or to the interstitial
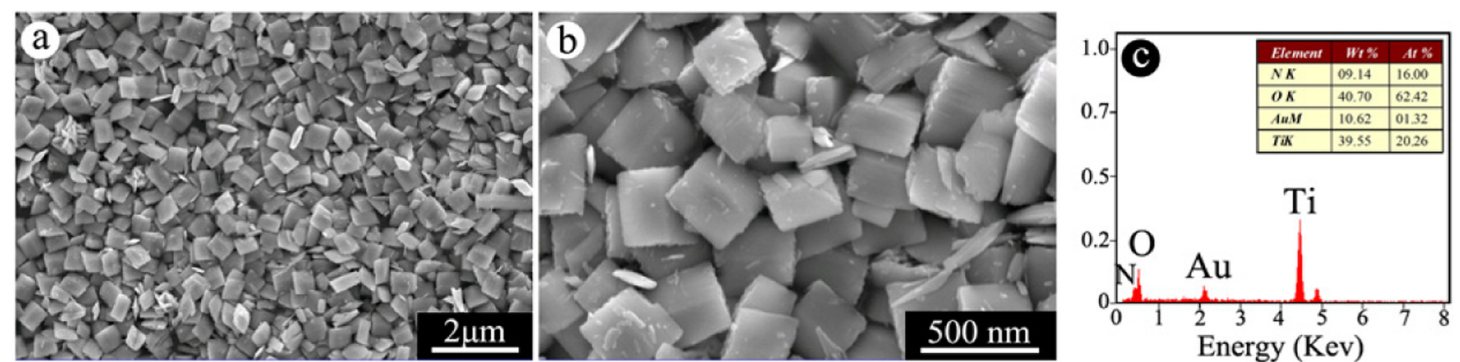

Figure 2. SEM images (a, b) and EDS (c) of $\mathrm{N}$-doped $\mathrm{TiO}_{2}$ microcuboids. 


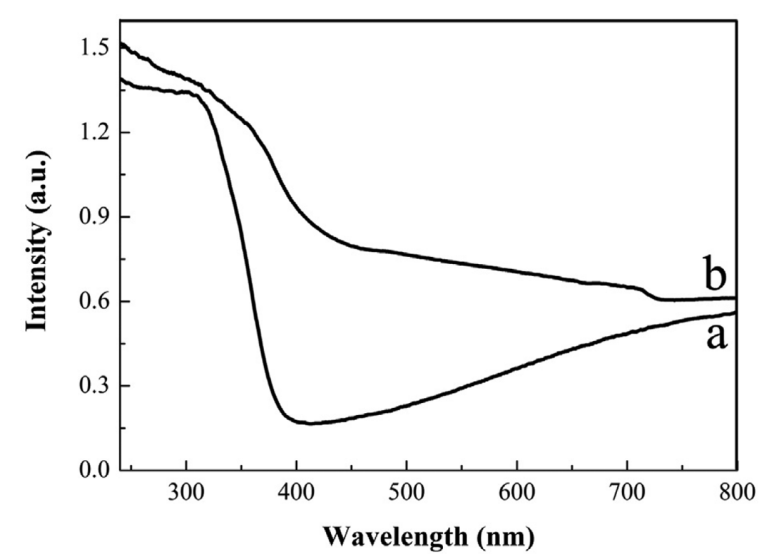

Figure 3. UV-vis spectra of $\mathrm{TiO}_{2}$ samples (a) and $\mathrm{N}$-doped $\mathrm{TiO}_{2}$ microcuboids (b).

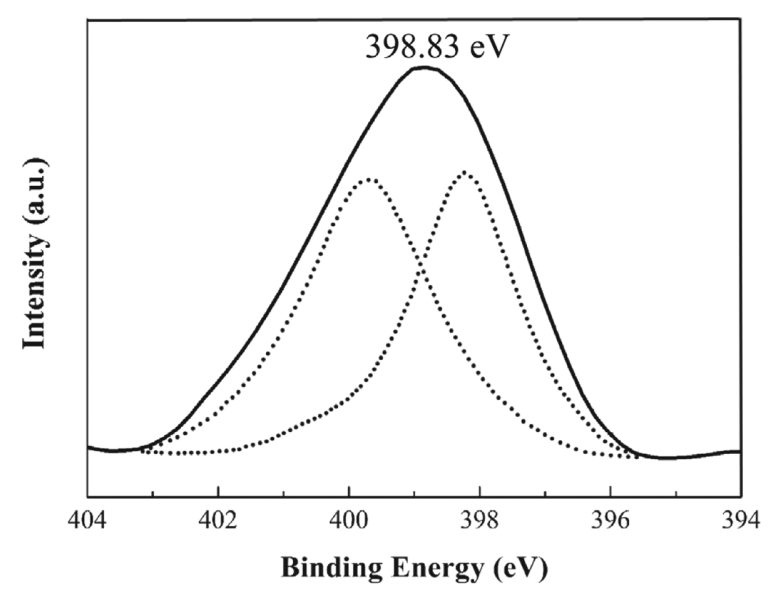

Figure 4. N1s XPS patterns spectrum of $\mathrm{N}$-doped $\mathrm{TiO}_{2}$ microcuboids.

introduction of nitride into the oxide lattice. ${ }^{16}$

Fig. 4 shows the N1s X-ray photoelectron spectroscopy spectrum of the $\mathrm{N}$-doped $\mathrm{TiO}_{2}$ microcuboids prepared by hydrothermal process. In general, the peak of N1s from the XPS spectrum is mostly found in the range of $396-404 \mathrm{eV}$. The N1s peak at $399.63 \mathrm{eV}$ can be attributed to the nitrogen in the form of a Ti-N-O linkage, and the low bonding energy component located at $398.23 \mathrm{eV}$ is generally known as the $\mathrm{N}$ atom replacing the oxygen atoms in the $\mathrm{TiO}_{2}$ crystal lattice to form an $\mathrm{N}-\mathrm{Ti}-\mathrm{N}$ bond. The relative atomic concentrations of $\mathrm{N}$ in the $\mathrm{TiO}_{2}$ sample is found to be 4.8 at.\% based on the XPS data.

The photocatalytic activity of the $\mathrm{TiO}_{2}$ samples and Ndoped $\mathrm{TiO}_{2}$ microcuboids was evaluated by measuring the rate of degradation of $\mathrm{RhB}$ under $\mathrm{UV}$ and visible light irradiation. Fig. 5(a) shows the degradation of RhB under UV irradiation for the two kinds of samples. After $150 \mathrm{~min}$ of UV irradiation, $87 \%$ of $\mathrm{RhB}$ is decomposed by $\mathrm{TiO}_{2}$ sam-
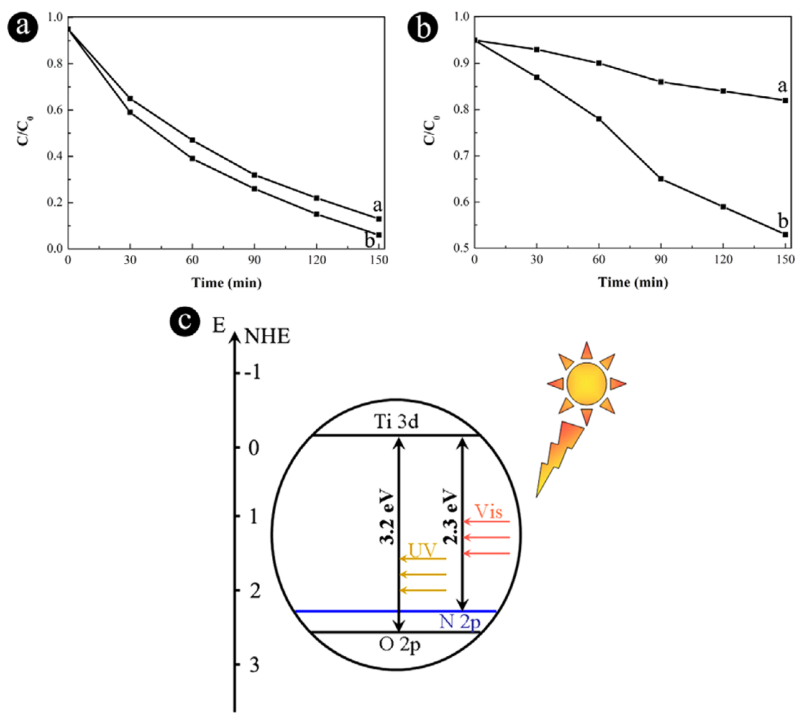

Figure 5. The photocatalytic degradation of $\mathrm{RhB}$ over $\mathrm{TiO}_{2}$ samples and $\mathrm{N}$-doped $\mathrm{TiO}_{2}$ microcuboids under UV (a) and visible light (b) irradiation. A schematic illustration of electron migration in $\mathrm{N}$-doped $\mathrm{TiO}_{2}$ microcuboids under $\mathrm{UV}$ and visible light irradiation (c).

ples, whereas $94 \%$ of $\mathrm{RhB}$ is decomposed by $\mathrm{N}$-doped $\mathrm{TiO}_{2}$ microcuboids. It indicates that $\mathrm{N}$-doped $\mathrm{TiO}_{2}$ microcuboids exhibits superior photocatalytic capability in degrading $\mathrm{RhB}$ dye under the UV irradiation, and the photocatalytic enhancement is mainly attributed to the effective separation of the photogenerated electron-holes pairs. Under UV light activation, the electrons of $\mathrm{TiO}_{2}$ are excited to the conduction band, the valence holes transferred from the valence band of $\mathrm{TiO}_{2}(\mathrm{O} 2 \mathrm{p})$ to that of $\mathrm{TiO}_{2}(\mathrm{~N} 2 \mathrm{p})$. Thus, electron-hole pairs are effectively separated, which leads to the high photocatalytic activity of $\mathrm{N}$-doped $\mathrm{TiO}_{2}$ microcuboids. However, under visible light activation, the $47 \%$ of $\mathrm{RhB}$ removal is obtained after $150 \mathrm{~min}$, while only $18 \%$ of the $\mathrm{RhB}$ removal is obtained in the photocatilytic process with the same illumination time. It is therefore concluded that $\mathrm{N}$-doping is very important to improve the visible light photocatalytic performance. It is speculated that the main reason is the narrowing of the band gap caused by the mixing of the $\mathrm{N} 2 \mathrm{p}$ and $\mathrm{O} 2 \mathrm{p}$ states, which resulted in high visible light photocatalytic activity.

\section{CONCLUSION}

In conclusion, $\mathrm{N}$-doped $\mathrm{TiO}_{2}$ microcuboids were successfully fabricated by a facile one-pot hydrothermal method. The structure, optical and photocatalytic properties of $\mathrm{N}$-doped $\mathrm{TiO}_{2}$ microcuboids were discussed in 
detail, and the novel $\mathrm{N}$-doped $\mathrm{TiO}_{2}$ microstructures greatly enhanced the visible light response and photocatalytic degradation of RhB. The enhanced photocatalytic properties can be attributed to the extended absorption in the visible light region and the effective separation of photogenerated carriers resulting from $\mathrm{N}$ doping in the $\mathrm{TiO}_{2}$ crystals. This new type of photocatalyst that can harness both UV and visible light presents a promising candidate for applications in photocatalysis.

Acknowledgments. This work was financially supported by the National Natural Science Foundation of China (No. 61235006). And the publication cost of this paper was supported by the Korean Chemical Society.

\section{REFERENCES}

1. Yao, F.; Sun, Y.; Tan, C. L.; Wei, S.; Zhang, X. J.; Hu, X. Y.; Fan, J. J. Korean Phys. Soc. 2011, 55, 932.

2. Chen, W. G.; Yuan, P. F.; Zhang, S.; Sun, Q.; Liang, E.; Jia, Y. Physica B 2012, 407, 1038.

3. Ebrahimi, R.; Tarhandeh, G.; Rafiey, S.; Narjabadi, M.; Khani, H. J. Korean Phys. Soc. 2011, 56, 92.

4. Zhao, H. M.; Wu, M. M.; Wang, Q.; Jena, P. Physica B
2011, 406, 4322.

5. Yan, X. M.; Kang, J. L.; Gao, L.; Xiong, L.; Mei, P. Appl. Surf. Sci. 2013, 265, 778.

6. Nakajima, T.; Lee, C. Y.; Yang, Y.; Schmuki, P. J. Mater. Chem. A 2013, 1, 1860.

7. Chen, K. S.; Feng, X. R.; Hu, R.; Li, Y. B.; Xie, K.; Li, Y.; Gu, H. S. J. Alloys Compd. 2013, 554, 72.

8. Liu, B. K.; Wang, D. J.; Zhang, Y.; Fan, H. M.; Lin, Y. H.; Jiang, T. F.; Xie, T. F. Dalton Trans. 2013, 42, 2232.

9. Dunnill, C. W.; Ansari, Z.; Kazas, A.; Perni, S.; Morgan, D. J.; Wilson, M.; Parkin, I. P. J. Mater. Chem. 2011, 21, 11854.

10. Ku, Y.; Chen, W. J.; Hou, W. M. Sustain. Environ. Res. 2013, 23, 15.

11. Wang, D. H.; Jia, L.; Wu, X. L.; Lu, L. Q.; Xu, A. W. Nanoscale 2012, 4, 576.

12. Wu, M. C.; Liao, H. C.; Cho, Y. C.; Tóth, G.; Chen, Y. F.; Su, W. F.; Kordás, K. J. Mater. Chem. A 2013, 1, 5715.

13. Yang, G. D.; Jiang, Z.; Shi, H. H.; Xiao, T. C.; Yan, Z. F. J. Mater. Chem. 2010, 20, 5301.

14. Pan, J. H.; Han, G.; Zhou, R.; Zhao, X. S. Chem. Commun. 2011, 47, 6942.

15. Gai, L. G.; Duan, X. Q.; Jiang, H. H.; Mei, Q. H.; Zhou, G. W.; Tian, Y.; Liu, H. Cryst. Eng. Commun. 2012, 14, 7662.

16. Li, M.; Zhang, J. Y.; Zhang, Y. Catal. Commun. 2012, 29, 175. 